\title{
Increasing incidence of base of tongue cancers from 2000 to 2010 due to HPV: the largest demographic study of 210 Danish patients
}

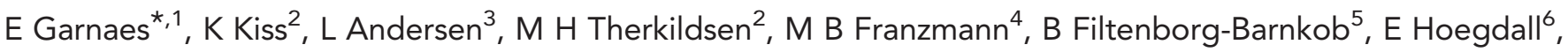
C B Lajer ${ }^{1}$, E Andersen ${ }^{7}$, L Specht ${ }^{8}$, L Joenson $^{9}$, K Frederiksen $^{10}$, L Friis-Hansen ${ }^{9}$, F C Nielsen ${ }^{9}$, S K Kjaer $^{11}$, B Norrild ${ }^{12}$ and $C$ von Buchwald ${ }^{1}$

${ }^{1}$ Department of Otorhinolaryngology, Head and Neck Surgery and Audiology, Rigshospitalet, University of Copenhagen, Copenhagen, Denmark; ${ }^{2}$ Department of Pathology, Rigshospitalet, University of Copenhagen, Copenhagen, Denmark; ${ }^{3}$ Department of Pathology, Roskilde Hospital, Roskilde, Denmark; ${ }^{4}$ Department of Pathology, Hvidovre Hospital, Hvidovre, Denmark; ${ }^{5}$ Department of Pathology, Naestved/Slagelse Hospital, Region of Zealand, Denmark; ${ }^{6}$ Department of Pathology, Herlev Hospital, University of Copenhagen, Copenhagen, Denmark; ${ }^{7}$ Department of Oncology, Herlev Hospital, University of Copenhagen, Copenhagen, Denmark; ${ }^{8}$ Department of Oncology, Rigshospitalet, University of Copenhagen, Copenhagen, Denmark; ${ }^{9}$ Center for Genomic Medicine, Rigshospitalet, University of Copenhagen, Copenhagen, Denmark; ${ }^{10}$ Department of Statistics, Bioinformatics and Registry, Danish Cancer Society Research Center, Copenhagen, Denmark; ${ }^{11}$ Department of Virus, Lifestyle and Genes, Danish Cancer Society Research Center and Department of Gynaecology, Rigshospitalet, University of Copenhagen, Copenhagen, Denmark and ${ }^{12}$ Institute of Cellular and Molecular Medicine, Panum Institute, University of Copenhagen, Copenhagen, Denmark

Background: We assessed the development in the number of new base of tongue squamous-cell carcinoma (BSCC) cases per year in eastern Denmark from 2000 to 2010 and whether HPV may explain any observable increased incidence.

Methods: We performed HPV DNA PCR and p16 immunohistochemistry analysis for all $(n=210)$ BSCCs registered in the Danish Head and Neck Cancer Group (DAHANCA) and the Danish Pathology Data Bank, and genotyped all HPV-positive specimens with amplicon-based next-generation sequencing.

Results: The overall crude incidence of BSCCs increased significantly (5.4\% per year) during the study period. This was explained by a significant increase in the number of HPV-positive BSCCs ( $8.1 \%$ per year), whereas the number of HPV-negative BSCCs did not increase significantly. The overall HPV prevalence was $51 \%$, with HPV16 as the predominant HPV type.

Conclusions: The increased number of HPV-positive BSCCs may explain the increasing incidence of BSCCs in eastern Denmark, 2000-2010.

HPV gives rise to a distinct clinical entity of oropharyngeal squamous-cell carcinomas (OPSCC) with a substantially better prognosis than HPV-negative OPSCC, often related to tobacco and alcohol consumption (Lajer et al, 2012). Although the incidence of OPSCC has been reported to be increasing in the Western world, including Denmark (Garnaes et al, 2014), a parallel decline in the

*Correspondence: E Garnaes; E-mail: emilie.nyberg.garnaes@rh.regionh.dk

Received 22 December 2014; revised 24 April 2015; accepted 5 May 2015; published online 4 June 2015

(c) 2015 Cancer Research UK. All rights reserved 0007-0920/15 
incidence of tobacco/alcohol-related head and neck cancers has been observed in most countries, consistent with reduced tobacco use (Sturgis and Cinciripini, 2007). Accordingly, we recently demonstrated that the increasing incidence of palatine tonsillar squamous-cell carcinoma (TSCC) in eastern Denmark from 2000 to 2010 may be attributed to an increased number of HPV-positive cases (Garnaes et al, 2014). The base of the tongue comprising the lingual tonsils has a tissue structure similar to the palatine tonsil, which has been associated with oropharyngeal HPV infection and cancer (Begum et al, 2005). However, few studies focused specifically on the epidemiology of HPV in base of tongue squamous-cell carcinomas (BSCCs), even though BSCC is the second most common OPSCC (after TSCC) in Denmark and other Western countries (Attner et al, 2010).

Previous studies reported highly variable BSCC HPV prevalence ranging from 2\% in Northern Spain, 1990-2009 (Rodrigo et al, 2014), to $68 \%$ in the USA, 1996-2007 (Stoler et al, 2013). According to two reports from Sweden, $40 \%$ and $75 \%$ of BSCC specimens were HPV-positive, 1970-2002 and 1998-2007, respectively (Dahlgren et al, 2004; Attner et al, 2010). These studies represented relatively small patient series, with the number of cases ranging from 25 to 108 (Dahlgren et al, 2004; Rodrigo et al, 2014). It is unclear whether the wide range in the reported HPV prevalence represents the true impact of HPV on BSCCs or if the deviations are caused by other factors, including variations in study time, geography and/or differences in the sensitivity and specificity of the HPV-detection techniques used (Gronhoj et al, 2014).

On the basis of this inconsistency, we included the largest, non-selected, consecutive cohort comprising all BSCC patients diagnosed in eastern Denmark during an 11-year study period (2000-2010). The aims were to explore whether the incidence of BSCCs increased from 2000 to 2010, whether HPV may explain the increase and to assess the HPV DNA prevalence and its association with gender and age. Furthermore, we examined the HPV genotypes in HPV-positive BSCCs and determined the correlation between HPV DNA PCR and p16 IHC.

\section{MATERIALS AND METHODS}

Patients and tumour samples. We identified all patients registered with base of tongue cancer in eastern Denmark from 2000 to 2010 in the Danish Head and Neck Cancer Group (DAHANCA) Database (www.dahanca.dk). We also linked our cohort to the Danish Pathology Data Bank using the personal identification codes as previously described (Garnaes et al, 2014). The geographical area of eastern Denmark contains $\sim 45 \%$ of the Danish population (Supplementary Figure 1), and BSCCs represent $\sim 20 \%$ of Danish OPSCCs (Johansen et al, 2000). The current study was conducted according to the Helsinki Declaration. It was approved by the Regional Scientific Ethical Committee (H-C-2008080) and the Danish Data Protection Agency.

Review of pathology and laboratory analysis. Specialized head and neck pathologists reviewed all histological tumour specimens and assessed p16 expression by IHC as described earlier (Garnaes et al, 2014). The handling of the tumour specimens, the DNA isolation and HPV DNA PCR analysis (GP5 + /GP6 + primers) were performed as previously reported (Garnaes et al, 2014).

We performed amplicon-based next-generation sequencing on all HPV DNA-positive samples. We prepared $50 \mathrm{ng}$ of DNA of each sample, and made four pools of 23 samples (pool 1-4) and one pool of 16 samples (pool 5). Each pool was ligated with a unique adaptor using the SPRIworks Fragment Library System (Beckman Coulter, Brea, CA, USA) and subsequently sequenced on the MiSeq (Illumina, San Diego, CA, USA) according to the manufacturer's protocol. We normalized pool 5, which only contained 16 samples, to 23 samples using an index of 23/16, and merged the reads from the five pools. The reads were imported into the CLC Main Workbench software (Qiagen, Hilden, Germany) for read mapping against HPV.

Statistical analysis. The statistical analyses were done as earlier described (Garnaes et al, 2014). Because of the limited study period, we used absolute numbers to analyse incidence.

\section{RESULTS}

Patient population. A total of 263 patients with base of tongue cancer were identified in eastern Denmark during 2000-2010 as registered in the DAHANCA database, and 252 patients (96\%) were also listed in the Danish Pathology Data Bank (Figure 1). We were able to retrieve the tumour specimens on 239 patients and 221 of these were recognised as squamous-cell carcinomas. Our final data set comprised 210 patients with specimens suitable for HPV detection. Patients included in the study were similar in terms of age, gender and year of diagnosis compared with those excluded (Supplementary Table 1). Overall, HPV DNA was present in $51 \%(108 / 210)$ of BSCCs.

Trends in BSCC incidence in eastern Denmark, 2000-2010. The total number of BSCC patients increased significantly from 2000 to 2010 (annual percentage change (APC), 5.4\%; 95\% CI, 1.0-10.1\%;

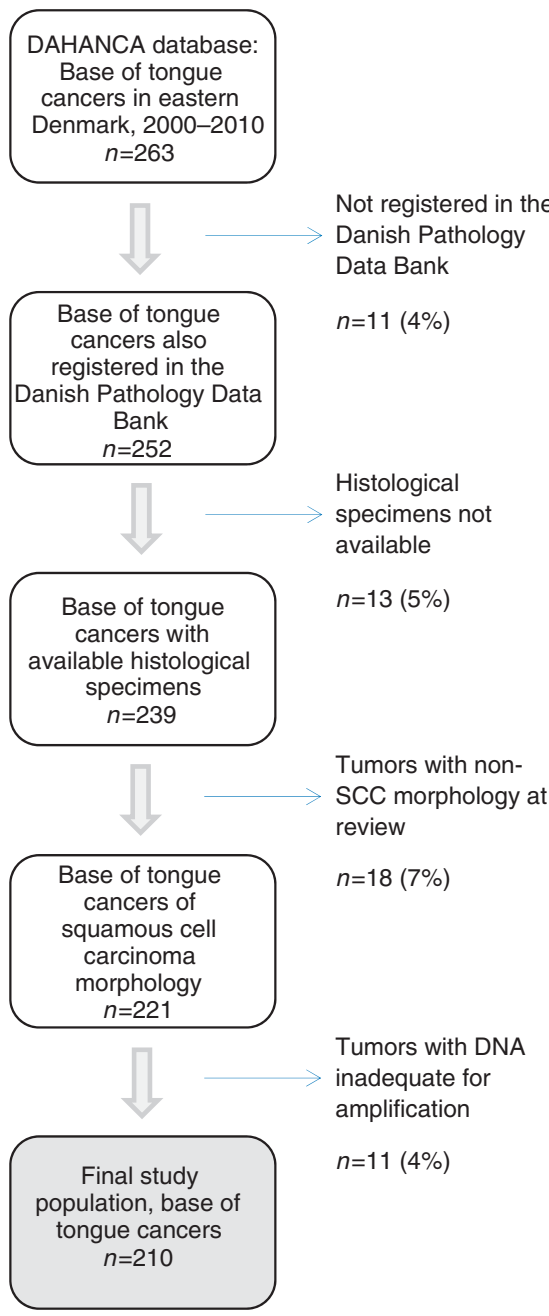

Figure 1. Overview of exclusions and final study population. Excluded patients are indicated as number of patients $(n)$ and percentage of total. 


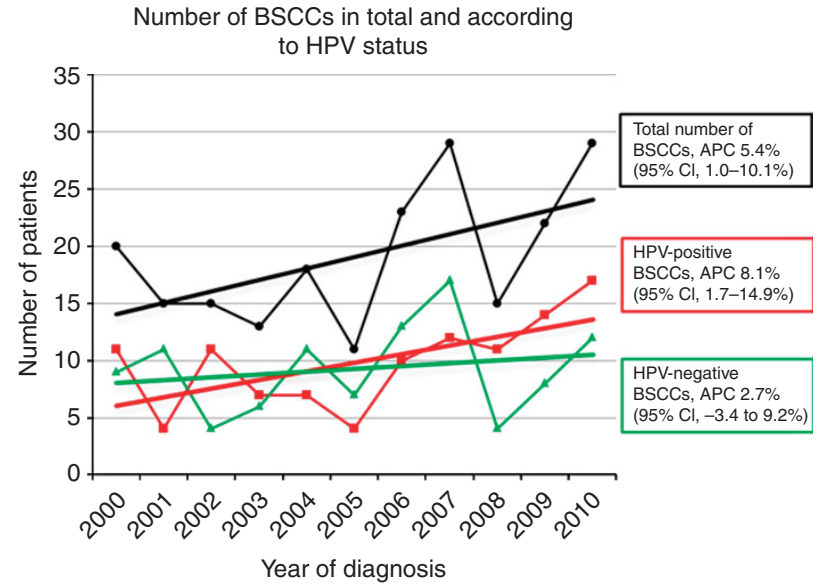

Figure 2. Number of total BSCCs according to HPV status. The black circles represent the total number of BSCCs per year; the black line is an estimated regression line showing a significant increase in the number of patients with BSCC (5.4\% per year). The red squares represent the number of patients with HPV-positive BSCCs per year; the red line is an estimated regression line showing a significant increase in the number of patients with HPV-positive BSCCs (8.1\% per year). The green triangles represent the number of patients with HPV-negative BSCCs per year; the green line is an estimated regression line showing no significant increase in the number of patients with HPV-negative BSCCs (2.7\% per year).

Abbreviations: $\mathrm{APC}=$ annual percentage change; $\mathrm{Cl}=$ confidence interval.

Figure 2). The number of HPV-positive patients also increased significantly during the study period (APC, 8.1\%; 95\% CI, $1.7-$ $14.9 \%)$. In contrast, the number of HPV-negative patients did not change significantly (APC, 2.7\%; 95\% CI, - 3.4-9.2\%).

HPV genotyping. We mapped a total of 7894496 reads against HPV of which 7576378 (96\%) reads were equivalent to HPV16, 185028 (2.3\%) reads represented HPV35, 72873 reads (0.9\%) corresponded to HPV45 and 60217 reads (0.8\%) were identified as HPV33.

Factors associated with HPV-positivity. We found no significant association of HPV prevalence with age, although patients $<60$ years had a higher HPV prevalence $(55 \%)$ than patients $\geqslant 60$ years $(48 \%$; odds ratio $(\mathrm{OR}) \geqslant 60$ vs $<60,1.46$; 95\% CI, 0.78-2.36; $P=0.27)$. Likewise, the HPV prevalence among female and male patients was similar ( $45 \%$ and $55 \%$, respectively; OR female vs male, 0.68 ; 95\% CI, $0.38-1.24 ; P=0.21$ ).

In total, $52 \%(110 / 210)$ of the specimens were p16 positive. HPV DNA PCR and p16 IHC, were in agreement in $88 \%$ of BSCCs (Table 1) $($ Kappa $=0.75 ; 95 \%$ CI, 0.67-0.85). The discordant

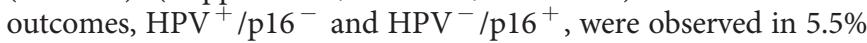
and $6.5 \%$ of BSCCs, respectively.

\section{DISCUSSION}

In the present study, we included 210 DAHANCA BSCC patients representing eastern Denmark from 2000 to 2010. It is the largest, non-selected, consecutive cohort of BSCCs to date, and it covers an area of $\sim 45 \%$ of the Danish population. We identified an overall HPV DNA prevalence of $51 \%$, and observed an increase in the number of HPV DNA-positive BSCCs, which may explain the increasing incidence of total BSCCs during the study period. To the best of our knowledge, this has not been reported previously.

The identified HPV DNA prevalence is lower than those reported in studies on BSCCs from Sweden and the USA (Attner et al, 2010; Stoler et al, 2013), and slightly lower than that observed in our TSCC study (Garnaes et al, 2014). Still, because of the size of
Table 1. Distribution of patients according to HPV DNA PCR and $\mathrm{p} 16 \mathrm{IHC}$

\begin{tabular}{l|c|c|}
\hline BSCCs & HPV-negative & HPV-positive \\
\hline Kappa: 0.75 (95\% Cl 0.67-0.85) & Number (\%) & Number (\%) \\
\hline p16 negative & $88(42 \%)$ & $12(5.5 \%)$ \\
\hline p16 positive & 14 (6.5\%) & $96(46 \%)$ \\
\hline $\begin{array}{l}\text { Abbreviation: Cl, confidence interval. The } 2 \times 2 \text { table shows the number of patients with the } \\
\text { corresponding HPV and p16 profile and the percentage of total, as well as the kappa value, } \\
\text { including the } 95 \% \mathrm{Cl} .\end{array}$
\end{tabular}

our study, the results are likely to be a more general representation of BSCC patients in Denmark.

We observed a trend towards an association of HPV prevalence with younger age; however, the association did not reach statistical significance. This may be related to the number of included patients. Nevertheless, our results add to the discussion on the relevance of male HPV vaccination in Denmark, given that HPV16 was the predominant HPV type in both BSCCs and TSCCs (Garnaes et al, 2014).

One of the advantages of our study is that we examined both the HPV DNA and the p16 status using validated HPV DNA PCR primers (Lajer et al, 2012) and a high cutoff for p16 expression by IHC. The majority $(96 / 108,89 \%)$ of HPV DNA-positive BSCCs were also p16 positive, implying an HPV-associated carcinogenesis. In contrast, some reports on the improved survival of HPVpositive OPSCCs have only used p16 as surrogate marker for HPV (Lassen et al, 2009), even though p16 overexpression is not specific to HPV (Rietbergen et al, 2014).

The current treatment regimen for OPSCC typically involves chemoradiotherapy without inclusion of HPV status. It is an intense therapy associated with considerable morbidity. A subgroup of HPVpositive BSCCs (and TSCCs) may not need such treatment why several clinical trials are investigating strategies for treatment deescalating. Furthermore, transoral robotic surgery may also represent a new, gentler treatment option. This highlights the importance of how to define HPV-positivity, and emphasizes the need for studies that focus on the effect of both HPV and p16 for the prognosis.

In conclusion, we found that the observed increase in the number of HPV-positive BSCCs may explain the increasing incidence of BSCC in eastern Denmark, 2000-2010. We found HPV DNA in $51 \%$ of BSCCs with HPV16 as the predominant HPV type and a good correlation between HPV DNA PCR and p16 IHC. Therefore, we suggest to measure both parameters and to include both TSCCs and BSCCs when estimating the number of HPV-positive OPSCCs.

\section{ACKNOWLEDGEMENTS}

We thank Ewa Futoma, Stine Oestergaard, Mette Moldaschl and Aseeba Ayub, Center for Genomic Medicine, Rigshospitalet, University of Copenhagen for technical assistance. This study was supported by the Research Foundation of Rigshospitalet (Rigshospitalets Forskningspuljer), the Villadsen Family Foundation, the Aragon Foundation, the Cancer Foundation of University of Copenhagen, the Danish Cancer Research Foundation, Aase and Ejnar Danielsen Foundation, Dagmar Marshall Foundation and Sanofi Pasteur MSD.

\section{CONFLICT OF INTEREST}

EG and $\mathrm{CvB}$ received research funding from Sanofi Pasteur MSD (unrestricted grant, 2012). EH received compensation for travel expenses to ASCO from Roche. CBL has received a fee for a lecture 
from Sanofi Pasteur MSD. LS has a consulting role and has received research funding from Takeda, Merck Serono and Boeringer Ingelheim. SKK received lecture fees, scientific advisory board fees and unrestricted institutional research grants from Merck and Sanofi Pasteur MSD. The remaining authors declare no conflict of interest.

\section{REFERENCES}

Attner P, Du J, Nasman A, Hammarstedt L, Ramqvist T, Lindholm J, Marklund L, Dalianis T, Munck-Wikland E (2010) The role of human papillomavirus in the increased incidence of base of tongue cancer. Int J Cancer 126(12): 2879-2884.

Begum S, Cao D, Gillison M, Zahurak M, Westra WH (2005) Tissue distribution of human papillomavirus 16 DNA integration in patients with tonsillar carcinoma. Clin Cancer Res 11(16): 5694-5699.

Dahlgren L, Dahlstrand HM, Lindquist D, Hogmo A, Bjornestal L, Lindholm J, Lundberg B, Dalianis T, Munck-Wikland E (2004) Human papillomavirus is more common in base of tongue than in mobile tongue cancer and is a favorable prognostic factor in base of tongue cancer patients. Int J Cancer 112(6): 1015-1019.

Garnaes E, Kiss K, Andersen L, Therkildsen MH, Franzmann MB, Filtenborg-Barnkob B, Hoegdall E, Krenk L, Josiassen M, Lajer CB, Specht L, Frederiksen K, Friis-Hansen L, Nielsen FC, Kjaer SK, Norrild B, von BC (2014) A high and increasing HPV prevalence in tonsillar cancers in Eastern Denmark, 2000-2010: the largest registry-based study to date. Int J Cancer 136(9): 2196-2203.

Gronhoj LC, Gyldenlove M, Jensen DH, Therkildsen MH, Kiss K, Norrild B, Konge L, von Buchwald C (2014) Correlation between human papillomavirus and p16 overexpression in oropharyngeal tumours: a systematic review. Br J Cancer 110(6): 1587-1594.
Johansen LV, Grau C, Overgaard J (2000) Squamous cell carcinoma of the oropharynx-an analysis of treatment results in 289 consecutive patients. Acta Oncol 39(8): 985-994.

Lajer CB, Garnaes E, Friis-Hansen L, Norrild B, Therkildsen MH, Glud M, Rossing M, Lajer H, Svane D, Skotte L, Specht L, Buchwald C, Nielsen FC (2012) The role of miRNAs in human papilloma virus (HPV)-associated cancers: bridging between HPV-related head and neck cancer and cervical cancer. Br J Cancer 106(9): 1526-1534.

Lassen P, Eriksen JG, Hamilton-Dutoit S, Tramm T, Alsner J, Overgaard J (2009) Effect of HPV-associated p16INK4A expression on response to radiotherapy and survival in squamous cell carcinoma of the head and neck. J Clin Oncol 27(12): 1992-1998.

Rietbergen MM, Snijders PJ, Beekzada D, Braakhuis BJ, Brink A, Heideman DA, Hesselink AT, Witte BI, Bloemena E, Baatenburg-de Jong RJ, Leemans CR, Brakenhoff RH (2014) Molecular characterization of p16-immunopositive but HPV DNA-negative oropharyngeal carcinomas. Int J Cancer 134(10): 2366-2372.

Rodrigo JP, Heideman DA, Garcia-Pedrero JM, Fresno MF, Brakenhoff RH, Diaz Molina JP, Snijders PJ, Hermsen MA (2014) Time trends in the prevalence of HPV in oropharyngeal squamous cell carcinomas in northern Spain (1990-2009). Int J Cancer 134(2): 487-492.

Stoler DL, Smaldino PJ, Darbary HK, Sullivan MA, Popat SR, Hicks Jr. WL, Merzianu M, Gaile DP, Anderson GR, Loree TR (2013) Human papillomavirus and tobacco use in tongue base cancers. Ear Nose Throat J 92(8): 372-380.

Sturgis EM, Cinciripini PM (2007) Trends in head and neck cancer incidence in relation to smoking prevalence: an emerging epidemic of human papillomavirus-associated cancers? Cancer 110(7): 1429-1435.

This work is published under the standard license to publish agreement. After 12 months the work will become freely available and the license terms will switch to a Creative Commons AttributionNonCommercial-Share Alike 4.0 Unported License

Supplementary Information accompanies this paper on British Journal of Cancer website (http://www.nature.com/bjc) 